\title{
Probing dynamical cortical gating of attention with concurrent TMS-EEG
}

Yuka O. Okazaki ${ }^{a}$, Yuji Mizuno ${ }^{\text {a, b }}$, Keiich Kitajo ${ }^{\text {a, c, d, * }}$

${ }^{\mathrm{a} C B S}$-TOYOTA Collaboration Center, RIKEN Center for Brain Science, 2-1 Hirosawa, Wako,

Saitama, 351-0198, Japan

${ }^{\mathrm{b}}$ Research Fellow of Japan Society for the Promotion of Science (JSPS), 5-3-1 Kojimachi,

Chiyoda-ku, 102-0083, Tokyo, Japan

${ }^{c}$ Division of Neural Dynamics, Department of System Neuroscience, National Institute for

Physiological Sciences, National Institutes of Natural Sciences, 38 Nishigonaka, Myodaiji, Okazaki,

Aichi, 444-8585, Japan

${ }^{\mathrm{d}}$ Department of Physiological Sciences, School of Life Science, The Graduate University for

Advanced Studies (SOKENDAI), 38 Nishigonaka, Myodaiji, Okazaki, Aichi, 444-8585, Japan.

Abbreviations: TR: task-relevant, TIR: task-irrelevant, CSD: current source density, TEP: TMS

evoked potential, TFR: time-frequency representations, AMI: alpha modulation index, ALI: alpha

lateralization index, lagPLV: lagged phase locking value, AEP: auditory evoked potential

* Corresponding author: Division of Neural Dynamics, Department of System Neuroscience,

National Institute for Physiological Sciences, National Institutes of Natural Sciences, 38

Nishigonaka, Myodaiji, Okazaki, Aichi, 444-8585, Japan

E-mail: kkitajo@nips.ac.jp 


\section{Highlights}

- Attention-regulated cortical excitability and effective connectivity were probed by TMS-EEG.

- Stronger TMS evoked potentials were observed in the task-relevant visual hemisphere.

- Strong effective connectivity between task-relevant visual cortex and other areas were found. 


\begin{abstract}
Background: Attention facilitates gating of information from the sending brain area to receiving

areas, with this being achieved by dynamical change in effective connectivity between cortices.
\end{abstract}

However, it is difficult to assess effective connectivity, which refers to the causal influence of one

cortical area on another.

Objective: The aim of this study is to directly probe the effective connectivity between cortical areas,

which is modulated by covertly shifted attention, excluding the thalamic influence.

Methods: Transcranial magnetic stimulation (TMS) was used to directly perturb the right retinotopic

visual cortex in task-relevant (TR) and task-irrelevant (TIR) networks, and the impact of this was

tracked to other areas by concurrent use of electroencephalography (EEG).

Results: Key finding was that TMS to the TR hemisphere led to a stronger evoked potential than did

stimulation to the TIR hemisphere. Moreover, stronger beta- and gamma-band effective

connectivities assessed as lagged phase synchronizations between stimulated areas and other areas

were observed when TMS was delivered to the TR area. These effects were more enhanced when

they preceded more prominent alpha lateralization, which is known to be associated with gating

information.

Conclusion: Our results indicate that attention-regulated cortical excitability and feedforward

dynamical effective connectivity can be probed by direct cortical stimulation to the TR or TIR area, 
thereby bypassing thalamic gating. These results bear out the idea that TMS-EEG could help to characterize changes in the functional architecture of brain networks that are required for rapid adaptation to the environment or due to brain diseases. 

aCC-BY-NC-ND 4.0 International license.

Keywords:

TMS-EEG, visual attention, cortical excitability, effective connectivity, alpha oscillations 


\section{Introduction}

In our daily lives, we are bombarded with sensory inputs far beyond our information processing

capacity. The covert direction of attention to specific parts of a visual scene allows processing

resources to be allocated to potentially relevant stimuli, typically through the modulation of local

neuronal excitability to attended and unattended sensory inputs [1-4].

However, attention can also change signal transmission, which is mediated by large-scale phase

synchronizations in neural activity between task-relevant regions [5-9]. In a study using a covert

visual attention paradigm, Doesburg and colleagues showed that gamma-band synchronization

between the contralateral occipital electrode and other electrodes increased during attention

maintenance $[5,10]$. Their results suggested that long-range gamma synchronization helps establish

a transient network that promotes information transmission from modality-specific cortical areas to

other cortical areas forming TR networks. On one hand, synchronizations between scalp electrodes

can be a result of spurious coupling that is actually driven by a common source or contamination

from volume conduction [11, 12], while on the other hand, the causal influence of one brain region

on others has been assessed by measuring effective connectivity $[13,14]$. The most straightforward

way to measure effective connectivity is to perturb a part of the brain network and observe how its

impact is transmitted to other sites. In human studies, this has been achieved by combining

transcranial magnetic stimulation (TMS) with EEG [13-19]. TMS-EEG has allowed demonstration 
of the dynamical properties of effective connectivity by showing how the propagation patterns of

TMS evoked potentials could be used to differentiate between sleep and wakeful states [13], as well

as the propagation of TMS-induced transient phase resetting of ongoing oscillations from visual to

motor areas [17]. In this study, we used such a perturbation approach to probe the dynamical nature

of cortical excitability and effective connectivity alterations between different attention conditions.

The spatiotemporal profiles of EEG responses were examined when TMS was applied to the right

V1/V2 of the task-relevant (TR) or task-irrelevant (TIR) hemisphere, depending on the attention

direction.

EEG-level phase synchronization between distant areas may be a plausible mechanism for

network communication [20,21]. If attention modulates effective connectivity depending on the task

at hand, we hypothesize that effective connectivity assessed as lagged phase synchronizations would

increase between TR areas when one of these areas is perturbed, while perturbation to TIR areas

would not induce such a prominent change in phase dynamics because effective connectivity would

be decreased. In addition, such modulation of effective connectivity may depend on the extent to

which alpha oscillations are modulated in the preparatory period [22].

\section{Materials and methods}

\section{Participants}

Twenty-two healthy right-handed participants ( 7 female and 15 male, mean age: $24.9 \pm 5.7$ [SD]) 
gave informed written consent for their participation in this study. The ethical committee of the

RIKEN Center for Brain Science approved this TMS-EEG study.

\section{Stimulus and task}

The TMS-EEG experiment consisted of six runs (three runs for real TMS, and three runs for

sham TMS), each containing 96 trials. Participants were seated $100 \mathrm{~cm}$ from a gamma corrected

LCD monitor (BenQ XL2420, $100 \mathrm{~Hz}$ refresh rate) and performed a cued spatial attention task

during both the real- and sham-TMS runs, which were performed in random order (Fig. 1a). A trial

began with an arrow cue (0.1 s) indicating the hemifield to which the participant should attend,

followed by an anticipatory interval of $1.2 \mathrm{~s}$. Subsequently, either a bilateral visual stimulus was

presented or TMS was applied to the right V1/V2 area (see EEG recordings and TMS), with the

order of these being randomly determined. In the visual stimulation trials, a target Gabor grating

(standard deviation of the Gaussian envelope, 0.18; spatial frequency, 2.5 cycles per degree [cpd];

contrast, $50 \%$; orientation, $\pm 2^{\circ}$ ) was presented in the cued hemifield together with a distractor

Gabor grating (orientation, $\pm 45^{\circ}$ ) in the other hemifield. These grating stimuli were presented for

$0.05 \mathrm{~s}$, followed by a $0.05 \mathrm{~s}$ bilateral backward mask stimulus (radial Gabor grating with the same

property as the target). Then, after $1 \mathrm{~s}$, participants were required to indicate whether the target

stimulus was tilted to the right or left by pressing the arrow keys with the index or middle finger of

their dominant right hand for the left or right orientation, respectively, with a response interval of $2 \mathrm{~s}$ 
being allowed for this, during which the color of a fixation cross changed from white to green. In the

TMS trials with no visual stimulation, the participants were asked to press freely either the left or

right arrow key during the response interval. Because it was unpredictable and counterbalanced as to

whether visual stimuli or TMS would be applied, the participants had to attend to the cued direction

in both conditions. Therefore, in TMS trials, either the TR or TIR right hemisphere could be

perturbed, depending on the attended direction (Fig. 1b). Stimulus delivery was controlled using

Psychtoolbox-3 [23-25].

EEG recordings and TMS

EEG (left earlobe reference; ground AFZ) signals were recorded from 63 scalp sites using

sintered $\mathrm{Ag} / \mathrm{AgCl}$ TMS-compatible electrodes mounted on a 10/10 system EasyCap (EASYCAP

Gmbh, Germany). Horizontal and vertical electrooculography (EOG) signals were continuously

recorded. The electrode impedance was kept below $10 \mathrm{k} \Omega$. The EEG and EOG signals were

amplified and recorded by a Brain Amp MR+ (Brain Products, Germany) system with a sampling

rate of $5 \mathrm{kHz}$. The electrode lead wires were arranged orthogonal to the TMS coil handle direction,

to reduce TMS-induced artifacts [26]. The TMS target site was located at the upper right V1/V2

according to the calcarine sulcus determined on each individual subject's MRI (mean Talairach

coordinates \pm SD: $11,-95 \pm 3,5$ ), and the TMS coil position was eventually near to the O2 electrode

for all participants. This TMS location approximately corresponds to the alpha modulated area (see 
Fig. 1c). The TMS coil and head position were continuously monitored using Brainsight TMS

(Rogue Research Inc., Canada), and kept within $5 \mathrm{~mm}$ of the initial position. For the sham

stimulation, the coil was rotated $90^{\circ}$ around the handle axis and spaced from the head using a $3 \mathrm{~cm}$

plastic cube [27]. Thus, the participant received some sensation of vibration caused by the TMS click,

without receiving direct cortical stimulation. Additionally, the click sound was attenuated by the

participant wearing earplugs and the delivery of white masking noise in all conditions. The

stimulation intensity achieving a 95\% active motor threshold in the right first dorsal interosseous

muscle was individually adjusted according to the distance between the TMS coil and targeted visual

cortex [28].

TMS and ocular artifact rejections

EEG data were analyzed using in-house developed scripts written in MATLAB (MathWorks,

Natick, USA) and FieldTrip [29]. The data were first segmented into $5 \mathrm{~s}$ epochs (3.5 s

pre-stimulation and $1.5 \mathrm{~s}$ post-stimulation), and then the epoched data were re-referenced offline to

the average of the right and left earlobe signals. TMS and ocular artifacts were rejected using the

following steps. First, the data samples were temporally smoothed using linearly interpolation (two

samples preceding and about 21 samples following the TMS onset, i.e., $4.3 \pm 0.08 \mathrm{~ms}$ ) to remove

excessive TMS artifacts. Second, epochs contaminated with eye movements and blinks in the

interval between -1.3 and $1.1 \mathrm{~s}$ were discarded according to the following criteria: horizontal EOG 
signals exceeding $\sim 50 \mu \mathrm{V}$, which approximately corresponded to the stimulus eccentricity $\left(6^{\circ}\right.$ visual angle), and vertical EOG exceeding $\sim 100 \mu \mathrm{V}$. Third, the exponential decay artifacts due to TMS were attenuated using independent component analysis (ICA) based on the method proposed by Korhonen and colleagues [30] (see also http://www.fieldtriptoolbox.org/tutorial/tms-eeg for a more practical application). ICs were excluded according to mean z-score values greater than 1.65 between 0 and $50 \mathrm{~ms}$, with the topography of the mixing matrix being confined within the stimulated region. For ocular artifacts, ICs correlating with the EOG $(r>0.2, p<0.05)$ and showing the typical topographical structure of saccadic eye movements and blinks were also excluded. Finally, temporal smoothing linear interpolation was again applied until $10 \mathrm{~ms}$ to remove residual artifact. Four participants for whom more than $45 \%$ of trials were lost after discarding epochs contaminated with ocular artifacts were excluded from further analysis. The artifact-removed EEG measures were then down-sampled to $500 \mathrm{~Hz}$. Current source density (CSD) transformation [31, 32] was applied to localize the activity and attenuate the effects of volume conduction.

TMS evoked potential (TEP)

To investigate the local cortical excitability, we took particular note of an initial cortical reaction in TEP. The preprocessed EEG epochs were bandpass filtered from 3 to $45 \mathrm{~Hz}$ using a fourth order Butterworth filter and averaged for each condition. The peak TEP components in the interval 0-0.2 s were identified. 


\section{Attentional alpha power modulation}

The instantaneous amplitude and phase were obtained using a wavelet transform with a center

frequency $f$, time $t$, and standard deviation $\sigma_{f}=4 f / m$ and $\sigma_{t}=m / 2 \pi f$ [33]. The constant $m$ was set to

3.Next the alpha modulation index was computed according to the following: $A M I_{c h}=\alpha_{c h}($ left cue $)-$

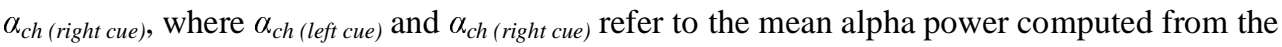

instantaneous amplitude for the left and right cued trials per electrode. For each participant, the most

positive electrode in the left hemisphere and the most negative electrode in the right hemisphere at

the time when the AMI was topographically maximized between -1 and $-0.1 \mathrm{~s}$ was chosen. The

grand averaged AMI and TFR of power from an individually selected electrode and topography are

shown in Figure 1c and d, respectively. Using selected electrodes, the alpha lateralization index was

computed as $A L I=\alpha_{\text {ipsilateral electrode }}-\alpha_{\text {contralateral electrode, }}$, which represents the contrast in alpha power between the ipsilateral and contralateral electrode in respect to the attention side. Then, the $50 \%$ of trials with the highest ALI were assigned to "high-ALI" trials, while the other half of trials were assigned to "low-ALI" trials. This meant that the alpha power in the TMS target area (right V1/V2) was relatively low in the left cue trials and high in the right cue trials in the high-ALI trials, while this contrast was marginal in the low-ALI trials.

\section{Inter-areal phase synchronization}

The effective connectivities between $\mathrm{O} 2$ and all other electrodes were assessed using the lagged 
phase locking value to determine the relationship between sending and receiving areas:

$$
\operatorname{lag} P L V=\left|\frac{1}{N} \sum_{k=1}^{N} \exp \left(j\left(\varphi_{O 2, k}\left(f, t_{r e f}\right)-\varphi_{Y, k}(f, t)\right)\right)\right|
$$

where $\varphi_{\mathrm{O} 2}$ and $\varphi_{\mathrm{Y}}$ are the instantaneous phase of frequency $f$ at electrode $\mathrm{O} 2$ and electrode $Y$ (all the other electrodes). $N$ denotes the number of trials, and the reference time was set to $t_{r e f}=0$, which was

the onset timing of the TMS. Thus, lagPLV evaluates the consistency of the phase difference

between electrode $\mathrm{O} 2$ (sending) at the TMS onset and the other electrodes (receiving) with a given

lag time.

\section{Statistics}

The TEP and lagPLV observed in the TR and TIR conditions were statistically evaluated. Note

that these were obtained by subtracting sham conditions to reduce the osteoconductive auditory

component.

For the initial cortical reaction to the TMS perturbation, the P20 component (average of five samples) of the $\mathrm{O} 2$ electrode was statistically assessed using a two-way ANOVA with task relevancy

(TR and TIR) and ALI (low ALI and high ALI) as fixed factors.

For lagPLV, a cluster-based permutation test [34] was used to verify whether visual spatial attention changed the degree of signal transmission from early visual cortex to other cortical areas. A 
cluster-based permutation test was used to determine whether the observed difference in the cluster

statistics between conditions was large enough to reject the null hypothesis, according to the

following procedures. First, all elements (i.e., 63 electrodes, $3-45 \mathrm{~Hz}, 0-0.5 \mathrm{~s}$ ) in the lagPLV

matrices between the left and right attention conditions in each low-ALI and high-ALI set were

compared using two-tailed paired $t$-tests. Then, contiguous negative and positive clusters in the

matrices were identified according to an uncorrected $p$-value threshold of $<0.05$, and the sums of the

$t$-values in clusters were calculated as cluster statistics. Second, to obtain the null distribution of the

test cluster statistic, the maximal cluster from matrices of randomly permuted two-condition labels

within participants was identified, with 500 iterations being used. Finally, using the 97.5 percentile

of the null distribution as the level of statistical significance, significant clusters of observed data

were identified.

\section{Results}

Pre-stimulus alpha power modulation by attention

The TFR of the power from individually selected electrodes was contrasted between left and

right cue trials and averaged over participants. The grand averaged TFR of the power indicated that

alpha power was continuously modulated in the interval between cue and TMS, approximately $1 \mathrm{~s}$

prior to the stimulus onset, and corresponding to the direction of attention (Fig. 1c). This result is

evidence that the participants were engaged in the attentional task directing their attention toward the 
visual hemifield of the cue side, even in those TMS trials without visual stimuli, although the behavioral performance was relatively low (correct rate mean \pm sd: $0.65 \pm 0.11$ ). Topographic representation of the mean AMI from -0.5 to 0 s indicates that the alpha power was clearly lateralized in the occipito-parietal electrodes, i.e., the alpha power in the ipsilateral hemisphere (TIR hemisphere) to the attention direction increased, and alpha power in the contralateral hemisphere (TIR hemisphere) decreased (Fig. 1d). Note that the TMS target (around O2) coincides with the region where alpha power was strongly modulated.

Alpha lateralization-dependent early TMS evoked potential

The TEP was calculated to examine the response to the TMS applied to the target area, with the alpha power just before stimulation being strongly modulated by attention. We identified several TEP components from the $\mathrm{O} 2$ electrodes (near to the stimulation site), including the P20, N50, P70, N100, and P120 components, in both the high-ALI and low-ALI trials (Fig. 2a). Cortical excitability influenced by the alpha oscillations should be reflected in an immediate response to the TMS perturbation, e.g., the P20. In high-ALI trials (left panel), the topographical map contrasting the left and right cue trials indicated that P20 showed a maximal difference at approximately the stimulation site, while the maximum differences in low-ALI trials were sparsely distributed. We quantified the effect of pre-stimulus alpha power on the P20 using a two-way ANOVA with the factors ALI type and task relevancy (Fig. 2b). This showed a main effect for task relevance $(F(1,17)=8.756, p=$ 
0.009), indicating that the P20 in the TR condition was larger than in the TIR condition, regardless of the amount of alpha lateralization. There was no significant main effect for $\operatorname{ALI}$ type $(F(1,17)=$ $1.099, p=0.309)$ or the interaction between factors $(F(1,17)=0.587, p=0.454)$. However, we further applied an ad hoc simple main-effect test to examine the difference between TR and TIR, doing this separately for the high-ALI and low-ALI trials. This revealed that the effect of task relevancy on the P20 component was significant in high-ALI trials $(F(1,17)=5.431, p=0.032)$, but not in low-ALI trials $(F(1,17)=1.398, p=0.253)$. These results indicate that the hemisphere with task relevance to the attention was in a highly excitable state, but that this was likely to depend on the degree of alpha power in that hemisphere.

Alpha lateralization-dependent effective connectivity

To probe how effective connectivity varies with attention, we assessed lagPLV to estimate the effective connectivity as a directional phase coupling between the sending and receiving areas. LagPLV makes it possible to know when and where the perturbed phase dynamics at one region affect other regions in the brain network. We compared lagPLVs between TR and TIR conditions and observed a significant difference only in the high-ALI trial where the alpha power of the stimulation region was low under the TR condition and high under the TIR condition. There were no significant differences in the low-ALI trials. To quantify the time-frequency characteristics of this significant difference, we examined the number of significant electrode pairs over time and frequency range 
(Fig. 3a). In the beta and low gamma band with a peak at $25 \mathrm{~Hz}$, the lagPLV of the TR condition was

found to be significantly stronger at more electrode pairs than in the TIR condition $(p<0.05)$. The

significant difference started at about $70 \mathrm{~ms}$, peaked at $114 \mathrm{~ms}$, and ended at about $150 \mathrm{~ms}$. These

results show that the TMS perturbation to the TR region more strongly and widely affected other

regions in comparison with the perturbation to the TIR region.

Next, we investigated the spatial extent of this difference in the beta $(16-30 \mathrm{~Hz})$ and gamma

bands $(31-45 \mathrm{~Hz})$. Figure $3 \mathrm{~b}$ shows a topographical map of the mean significant $t$-values identified

by the cluster-based permutation test. For the difference between the TR and TIR conditions in the

beta-band lagPLV, the effect of TMS perturbation on the TR region was confined to the

temporal-parietal region until about $100 \mathrm{~ms}$, and then to the frontal region. For the gamma-band

lagPLV, the difference was first observed in the frontal region contralateral to the stimulated site, and

then moved to the ipsilateral frontal region. These results suggest that networks between

short-distance TR areas are established with beta-band oscillations and that the chained response was

triggered by a local stimulation, whereas long distance networks may be established with

gamma-band oscillations.

\section{Discussion}

\section{Probing cortical excitability}

It is generally agreed that directing attention toward a specific location in the visual field 
facilitates perception and retinotopic neural responses to subsequently presented target stimuli at the attended location $[35,36]$. However, given the evidence from human fMRI studies that attention modulates activity in the lateral geniculate nucleus $[37,38]$, the enhancement of visual evoked activity in the striate $[36,39]$ and extrastriate $[40,41]$ cortices probably reflects amplified afferent inputs through thalamic gating. Thus, it is hard to separate the contribution from the thalamus when evaluating top-down modulation on the neocortex. Bestmann and colleagues tested this issue by direct cortical stimulation of the visual cortex, so that the visual stimulus did not pass through the retinogeniculate pathway [42]. They showed that spatial attention facilitates awareness of phosphenes induced by TMS, although there is still controversy over the relationship between initial sensory responses and behavioral perception.

In an analogous spatial attention paradigm, we observed neural responses to TMS in early visual cortex, instead of phosphene perception. In the current study, the direct neural responses at a very early latency, i.e., P20, were modulated by the direction of attention and its associated alpha power modulation preceding the neural responses. These findings are compatible with a report by Herring and colleagues [43], although in their study, the earliest attentional modulation occurred in the N40 TEP component, rather than in P20. N40 may reflect inhibitory feedback in response to TMS-induced cortical excitation, rather than the initial excitation itself [44]. A plausible explanation for the difference in timing of the attentional effect on the early TEP component may be partly 
related to the stimulated area. We confined the TMS target area to the right V1/V2, while Herring et al. defined a target area to ensure retinotopic phosphene perception. Given that it has been reported that stimulation sites generating retinotopic phosphenes are situated at V2 and V3 [45], the TEP components observed in Herring's study might be a direct response from the extrastriate cortex, rather than the striate cortex. Such a hierarchical difference may lead to a difference in timing. In summary, these results provide evidence that the cortico-cortical top-down influence on the excitability of early visual cortices can be regulated in parallel with thalamic gating. Moreover, it seems that a large alpha lateralization emphasizes this attentional effect, i.e., the smaller P20 can be accompanied by large-power alpha oscillations in the TMS target site, and vice versa.

\section{Probing cortical effective connectivity}

The function of attention is not only to change local neural excitability; it must also facilitate dynamical interactions between different areas, depending on the task at hand. This could be achieved by flexible control of effective connectivity mediated by phase synchronization of neural oscillations at the regions sending and receiving information. Doesburg and colleagues showed that TR early visual cortex and other regions were coupled with gamma-band phase synchronization during covertly sustained attention [10]. To demonstrate that such phase synchronization reflects the directional network for signal transmission modulated by attention, we locally perturbed either contralateral or ipsilateral visual cortex during covert attention. In direct perturbation approaches, 
effective connectivity has been evaluated by measuring the propagation of evoked activity $[13,15]$.

Although the validity of the lagPLV used here to investigate the effective connectivity is comparable

to that of the techniques used in these previously mentioned studies, the lagPLV with TMS

perturbation is more focused on directional interactions. When waves originating in the sending area

reach the receiving area, the phase difference between these areas becomes constant at that time; that

is, it becomes synchronized, but with a certain delay time. We found that the waves in the right early

visual area propagated widely and reached to the other cortical areas at certain delay time in the TR

condition, while it seemed to be suppressed in the TIR condition. These results suggest that cortical

gating of the feedforward input is achieved by regulating the effective connectivity in the phase

dynamics between cortical areas. Importantly, it should be noted that successful gating depended on

the degree of alpha lateralization caused by attention. Although the mechanism behind the alpha

contribution and its source is still unknown, the key role in generating the alpha oscillations may be

played by inhibitory GABAergic neurons [46-48]. In a model to test the cortical gating mechanism

to TMS evoked activity during slow wave sleep, increased GABA release from local inhibitory

neurons in the cortex was effective in reducing the propagation of evoked activity [49]. Thus, the

increased effective connectivity in the present study may be mediated by a GABAergic inhibitory

feedback in the neocortex or thalamus. We recommend that studies employing magnetic resonance

spectroscopy should be performed to reveal the roles of GABA release. 
Effect of the auditory artifact from the TMS click sound

In general, a limitation of TMS research is that the TMS pulse is accompanied by a click sound

of about 100 to $120 \mathrm{~dB}$ [50], as well as the introduction of several electromagnetic and/or muscle

artifacts. The click sound contaminates part of the TEP with an auditory evoked potential (AEP)

[51-53].We used a combination of earplugs and white noise to mask the click sound, and placed a

thin layer of foam between the TMS coil and the EEG cap to attenuate bone conduction of sound [13,

53-55]. In addition, we arranged the electrode leads to minimize electromagnetic artifacts during the

experiment [26], and attenuated any such artifacts using offline ICA analysis [30, 43]. If phase shifts

are caused by an AEP and/or TMS artifacts, we should acknowledge that the lagPLV may spuriously

increase, but this would not explain our results. Because the effects of these artifacts should be

identical across different attention conditions and we always made comparisons between conditions

with sham subtraction, we believe that the modulated lagPLV is associated with dynamical gating of

cortical information processing. Nevertheless, it should be noted that further developments in more

realistic sham stimulation [56-59] and electrode referencing methods [60] are awaited to allow the

TMS-EEG community to maximize the direct effects of TMS on cortical responses.

\section{Conclusions}

By directly stimulating TR or TIR areas, we succeeded in probing attention-regulated cortical

excitability and feedforward effective connectivity in the phase dynamics between cortical areas. 
This method provides evidence that attentional top-down control not only coordinates the sensory

input to the cortex by thalamic gating, but also changes the cortical excitability and effective

connectivity that mediates cortical gating between TR regions. The observation of perturbation

effects caused by direct use of TMS-EEG will allow the characterization of excitability and effective connectivity changes according to the task at hand as well as changes due to diseases which impair

networks such as stroke [61].

\section{Conflict of Interest Statement}

Keiichi Kitajo discloses potential conflict of interest with TOYOTA Motor Corporation for the

support of a research grant.

\section{Acknowledgments}

This study was supported by JST PRESTO, MEXT Grants-in-Aid for Scientific Research 26282169

and 15H05877, Grant-in-Aid for JSPS Fellows (26-8352), and a research grant from TOYOTA

Motor Corporation. We are grateful to Yoko Noguchi for help with TMS-EEG recording. 
Figure 1 (a) Experimental paradigm. The trial started with a cue indicating which hemifield to

attend to, and after $1.3 \mathrm{~s}$, TMS was applied to the right visual cortex without presentation of a visual

stimulus. To guarantee the participant's attention to the cued hemifield, trials requiring a response to

the target Gabor orientation in the cued hemifield were randomly introduced. The target Gabor

stimulus $\left( \pm 2^{\circ}\right.$ oriented $)$ in the cued side was always presented with a distractor stimulus $\left( \pm 45^{\circ}\right.$

oriented) on the opposite side. Participants were unable to predict whether a visual stimulus or TMS

would be applied, so they needed to follow the cued instructions. (b) A schematic figure for the

attention-dependent conditions. The right visual cortex where TMS was applied becomes the

task-relevant hemisphere in the left attention trials, while it becomes the task-irrelevant hemisphere

in the right attention trials. (c) Grand averaged time-frequency representations of the AMI (left cue -

right cue) in the left and right electrode. (d) Grand averaged topographic AMI (8-12 Hz) for the time

range from -0.5 to $0 \mathrm{~s}$. The white dot indicates the electrode position near the TMS coil.

Figure 2 Attentional modulation in TMS evoked potentials. (a) TEPs from $\mathrm{O} 2$ at the site of

stimulation for the high-ALI and low-ALI trials. Topographic differences between the left and right

cue trials are shown for each TEP component. (b) The first response to TMS, i.e., P20, was

significantly larger in the task-relevant condition with high alpha power modulation (high-ALI trials)

than in the task-irrelevant condition. The same contrasts with low-ALI trials were negligible. 
Figure 3 Attentional modulation in effective connectivity from V1/V2 to other areas. (a) A

time-frequency profile of the number of significant lagPLVs in the comparison between the

task-relevant (TR) and task-irrelevant (TIR) conditions indicates that large areas are synchronized in

the beta and gamma bands. This significant difference was observed only in high-ALI trials (right

panel). (b) A spatiotemporal profile of the significant difference between TR and TIR in high-ALI

trials shows that TMS perturbation on the task-relevant area has strong impacts on other cortical

areas, e.g., parietal and frontal regions. 
bioRxiv preprint doi: https://doi.org/10.1101/615708; this version posted April 23, 2019. The copyright holder for this preprint (which was not certified by peer review) is the author/funder, who has granted bioRxiv a license to display the preprint in perpetuity. It is made available under aCC-BY-NC-ND 4.0 International license.

\section{Figure 1}

a

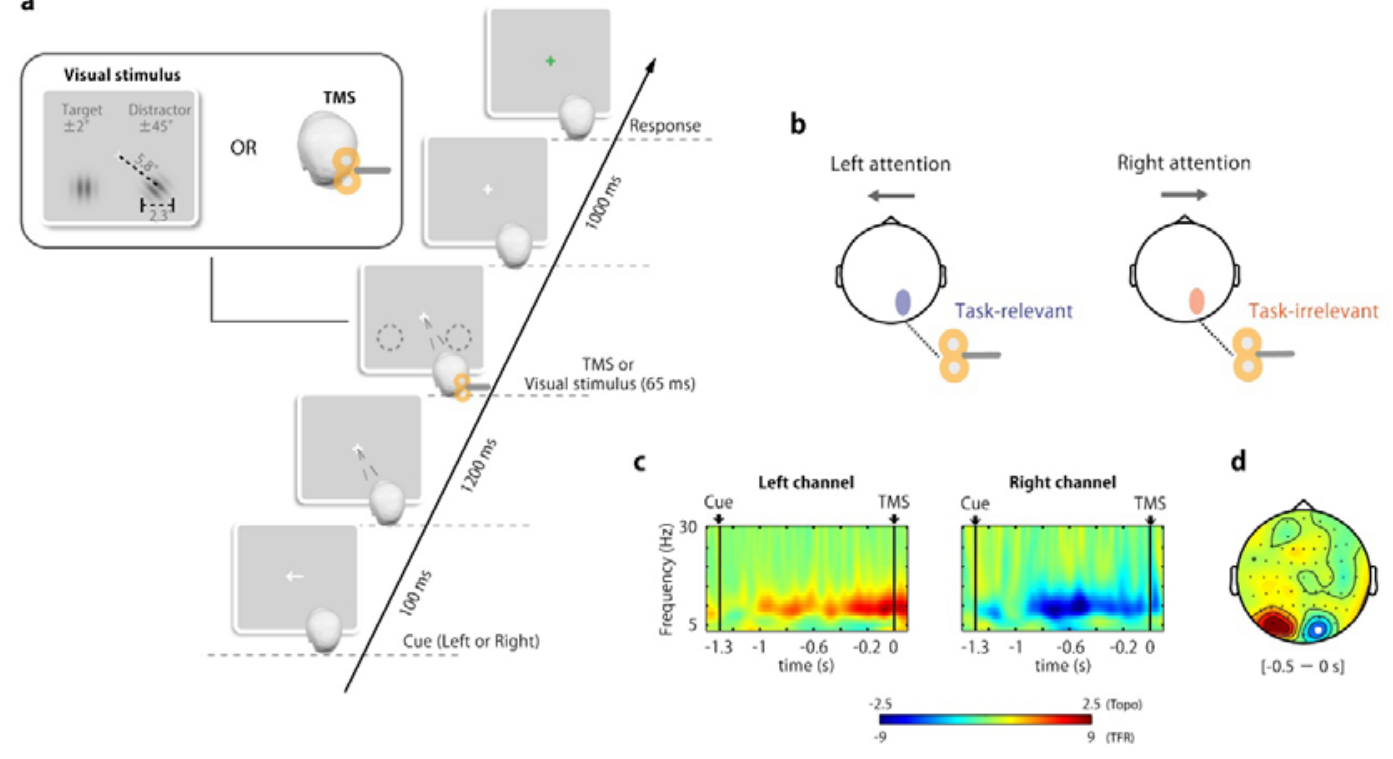


bioRxiv preprint doi: https://doi.org/10.1101/615708- this version posted April 23 2019. The copyright holder for this preprint (which was not certified by peer review) is the author/funder, who has granted bioRxiv a license to display the preprint in perpetuity. It is made available under aCC-BY-NC-ND 4.0 International license.

Figure 2

a

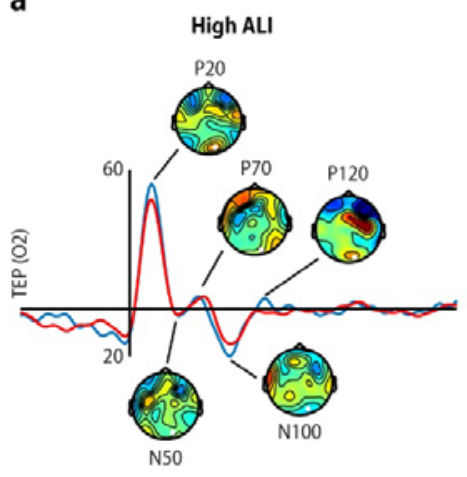

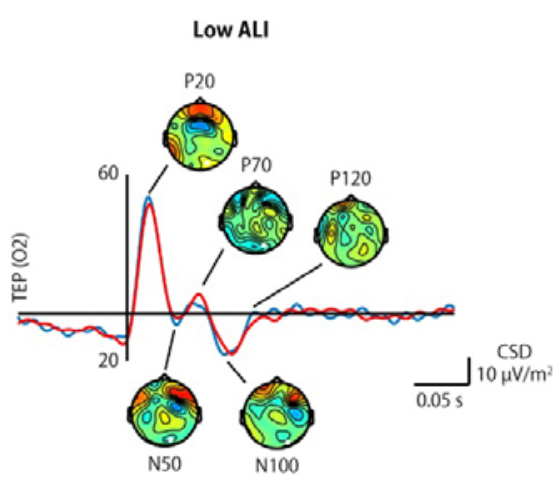

b

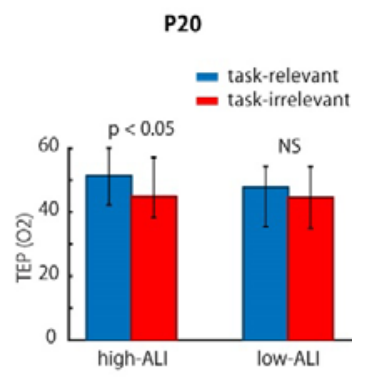

Figure 3

a

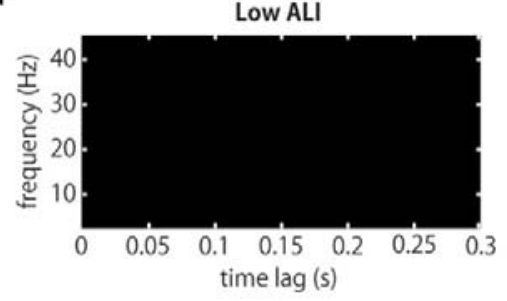

High ALI

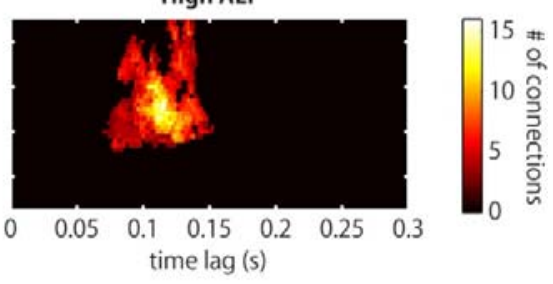

b

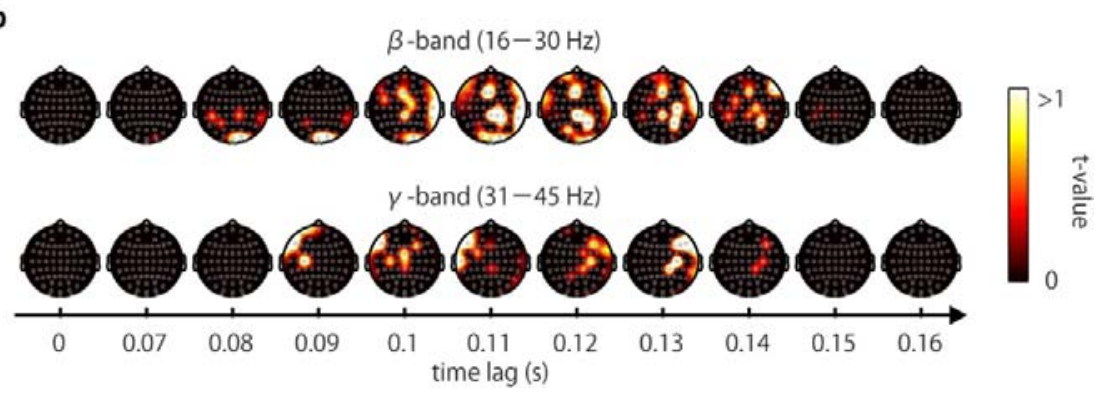


References

[1] Desimone R, Duncan J. Neural mechanisms of selective visual attention. Annu Rev Neurosci 1995;18:193-222.

[2] Lavie N, Tsal Y. Perceptual load as a major determinant of the locus of selection in visual attention. Percept Psychophys 1994;56(2):183-97.

[3] Rees G, Frith CD, Lavie N. Modulating irrelevant motion perception by varying attentional load in an unrelated task. Science 1997;278(5343):1616-9.

[4] VanRullen R, Koch C. Competition and selection during visual processing of natural scenes and objects. J Vision 2003;3(1):75-85.

[5] Doesburg SM, Green JJ, McDonald JJ, Ward LM. From local inhibition to long-range integration: A functional dissociation of alpha-band synchronization across cortical scales in visuospatial attention. Brain Res 2009;1303:97-110.

[6] Gross J, Schmitz F, Schnitzler I, Kessler K, Shapiro K, Hommel B, et al. Modulation of long-range neural synchrony reflects temporal limitations of visual attention in humans. Proc Natl Acad Sci USA 2004;101(35):13050-5.

[7] Zhou H, Schafer RJ, Desimone R. Pulvinar-cortex interactions in vision and attention. Neuron 2016;89(1):209-20.

[8] Daitch AL, Sharma M, Roland JL, Astafiev SV, Bundy DT, Gaona CM, et al. Frequency-specific mechanism links human brain networks for spatial attention. Proc Natl Acad Sci USA 2013;110(48):19585-90.

[9] Bosman CA, Schoffelen JM, Brunet N, Oostenveld R, Bastos AM, Womelsdorf T, et al. Attentional stimulus selection through selective synchronization between monkey visual areas. Neuron 2012;75(5):875-88.

[10] Doesburg SM, Roggeveen AB, Kitajo K, Ward LM. Large-scale gamma-band phase synchronization and selective attention. Cereb Cortex 2008;18(2):386-96.

[11] Kitajo K, Okazaki YO. TMS-EEG for probing distinct modes of neural dynamics in the human brain. Adv Cogn Neurodyn 2016;5:211-6.

[12] Palva S, Palva JM. Discovering oscillatory interaction networks with M/EEG: Challenges and breakthroughs. Trends Cogn Sci 2012;16(4):219-30.

[13] Massimini M, Ferrarelli F, Huber R, Esser SK, Singh H, Tononi G. Breakdown of cortical effective connectivity during sleep. Science 2005;309(5744):2228-32.

[14] Massimini M, Ferrarelli F, Esser SK, Riedner BA, Huber R, Murphy M, et al. Triggering sleep slow waves by transcranial magnetic stimulation. Proc Natl Acad Sci U S A 2007;104(20):8496-501.

[15] Ilmoniemi RJ, Virtanen J, Ruohonen J, Karhu J, Aronen HJ, Naatanen R, et al. Neuronal responses to magnetic stimulation reveal cortical reactivity and connectivity. Neuroreport 
1997;8(16):3537-40.

[16] Massimini M, Ferrarelli F, Murphy M, Huber R, Riedner B, Casarotto S, et al. Cortical reactivity and effective connectivity during rem sleep in humans. Cogn Neurosci 2010;1(3):176-83.

[17] Kawasaki M, Uno Y, Mori J, Kobata K, Kitajo K. Transcranial magnetic stimulation-induced global propagation of transient phase resetting associated with directional information flow. Front Hum Neurosci 2014;8:173.

[18] Morishima Y, Akaishi R, Yamada Y, Okuda J, Toma K, Sakai K. Task-specific signal transmission from prefrontal cortex in visual selective attention. Nat Neurosci 2009;12(1):85-91.

[19] Miyauchi E, Kitajo K, Kawasaki M. TMS-induced theta phase synchrony reveals a bottom-up network in working memory. Neurosci Lett 2016;622:10-4.

[20] Varela F, Lachaux JP, Rodriguez E, Martinerie J. The brainweb: Phase synchronization and large-scale integration. Nat Rev Neurosci 2001;2(4):229-39.

[21] Fries P. A mechanism for cognitive dynamics: Neuronal communication through neuronal coherence. Trends Cogn Sci 2005;9(10):474-80.

[22] Jensen O, Mazaheri A. Shaping functional architecture by oscillatory alpha activity: Gating by inhibition. Front Hum Neurosci 2010;4:186.

[23] Brainard DH. The psychophysics toolbox. Spat Vis 1997;10(4):433-6.

[24] Kleiner M, Brainard, D., Pelli, D., Ingling, A., Murray, R., Broussard, C. What's new in psychtoolbox-3. Perception 2007;36(14):1-16.

[25] Pelli DG. The videotoolbox software for visual psychophysics: Transforming numbers into movies. Spat Vis 1997;10(4):437-42.

[26] Sekiguchi H, Takeuchi S, Kadota H, Kohno Y, Nakajima Y. TMS-induced artifacts on EEG can be reduced by rearrangement of the electrode's lead wire before recording. Clin Neurophysiol 2011;122(5):984-90.

[27] Esser SK, Huber R, Massimini M, Peterson MJ, Ferrarelli F, Tononi G. A direct demonstration of cortical ltp in humans: A combined TMS/EEG study. Brain Res Bull 2006;69(1):86-94.

[28] Stokes MG, Chambers CD, Gould IC, Henderson TR, Janko NE, Allen NB, et al. Simple metric for scaling motor threshold based on scalp-cortex distance: Application to studies using transcranial magnetic stimulation. J Neurophysiol 2005;94(6):4520-7.

[29] Oostenveld R, Fries P, Maris E, Schoffelen JM. Fieldtrip: Open source software for advanced analysis of MEG, EEG, and invasive electrophysiological data. Comput Intell Neurosci 2011;2011:156869.

[30] Korhonen RJ, Hernandez-Pavon JC, Metsomaa J, Maki H, Ilmoniemi RJ, Sarvas J. Removal of large muscle artifacts from transcranial magnetic stimulation-evoked EEG by independent component analysis. Med Biol Eng Comput 2011;49(4):397-407.

[31] Kayser J, Tenke CE. Principal components analysis of laplacian waveforms as a generic 
method for identifying ERP generator patterns: II. Adequacy of low-density estimates. Clin Neurophysiol 2006;117(2):369-80.

[32] Kayser J, Tenke CE, Gates NA, Kroppmann CJ, Gil RB, Bruder GE. ERP/CSD indices of impaired verbal working memory subprocesses in schizophrenia. Psychophysiology 2006;43(3):237-52.

[33] Lachaux JP, Rodriguez E, Van Quyen ML, Lutz A, Martinerie J, Varela FJ. Studying single-trials of phase synchronous activity in the brain. Int J Bifurc Chaos 2000;10(10):2429-39.

[34] Maris E, Oostenveld R. Nonparametric statistical testing of EEG- and MEG-data. J Neurosci Methods 2007;164(1):177-90.

[35] Corbetta M, Shulman GL. Control of goal-directed and stimulus-driven attention in the brain. Nat Rev Neurosci 2002;3(3):201-15.

[36] Poghosyan V, Ioannides AA. Attention modulates earliest responses in the primary auditory and visual cortices. Neuron 2008;58(5):802-13.

[37] O'Connor DH, Fukui MM, Pinsk MA, Kastner S. Attention modulates responses in the human lateral geniculate nucleus. Nat Neurosci 2002;5(11):1203-9.

[38] McAlonan K, Cavanaugh J, Wurtz RH. Guarding the gateway to cortex with attention in visual thalamus. Nature 2008;456(7220):391-4.

[39] Di Russo F, Martinez A, Hillyard SA. Source analysis of event-related cortical activity during visuo-spatial attention. Cereb Cortex 2003;13(5):486-99.

[40] Woldorff MG, Liotti M, Seabolt M, Busse L, Lancaster JL, Fox PT. The temporal dynamics of the effects in occipital cortex of visual-spatial selective attention. Brain Res Cogn Brain Res 2002;15(1):1-15.

[41] Martinez A, Anllo-Vento L, Sereno MI, Frank LR, Buxton RB, Dubowitz DJ, et al. Involvement of striate and extrastriate visual cortical areas in spatial attention. Nat Neurosci 1999;2(4):364-9.

[42] Bestmann S, Ruff CC, Blakemore C, Driver J, Thilo KV. Spatial attention changes excitability of human visual cortex to direct stimulation. Curr Biol 2007;17(2):134-9.

[43] Herring JD, Thut G, Jensen O, Bergmann TO. Attention modulates TMS-locked alpha oscillations in the visual cortex. J Neurosci 2015;35(43):14435-47.

[44] Premoli I, Castellanos N, Rivolta D, Belardinelli P, Bajo R, Zipser C, et al. TMS-EEG signatures of gabaergic neurotransmission in the human cortex. J Neurosci 2014;34(16):5603-12.

[45] Kammer T, Beck S, Erb M, Grodd W. The influence of current direction on phosphene thresholds evoked by transcranial magnetic stimulation. Clin Neurophysiol 2001;112(11):2015-21.

[46] Lorincz ML, Kekesi KA, Juhasz G, Crunelli V, Hughes SW. Temporal framing of thalamic relay-mode firing by phasic inhibition during the alpha rhythm. Neuron 2009;63(5):683-96.

[47] Lorincz ML, Crunelli V, Hughes SW. Cellular dynamics of cholinergically induced alpha (8-13 hz) rhythms in sensory thalamic nuclei in vitro. J Neurosci 2008;28(3):660-71. 
[48] Hughes SW, Crunelli V. Thalamic mechanisms of EEG alpha rhythms and their pathological implications. Neuroscientist 2005;11(4):357-72.

[49] Esser SK, Hill S, Tononi G. Breakdown of effective connectivity during slow wave sleep: Investigating the mechanism underlying a cortical gate using large-scale modeling. J Neurophysiol 2009;102(4):2096-111.

[50] Starck J, Rimpilainen I, Pyykko I, Esko T. The noise level in magnetic stimulation. Scand Audiol 1996;25(4):223-6.

[51] Ilmoniemi RJ, Kicic D. Methodology for combined TMS and EEG. Brain Topogr 2010;22(4):233-48.

[52] Nikouline V, Ruohonen J, Ilmoniemi RJ. The role of the coil click in TMS assessed with simultaneous EEG. Clin Neurophysiol 1999;110(8):1325-8.

[53] ter Braack EM, de Jonge B, van Putten MJ. Reduction of TMS induced artifacts in EEG using principal component analysis. IEEE Trans Neural Syst Rehabil Eng 2013;21(3):376-82.

[54] Stokes MG, Chambers CD, Gould IC, English T, McNaught E, McDonald O, et al. Distance-adjusted motor threshold for transcranial magnetic stimulation. Clin Neurophysiol 2007;118(7):1617-25.

[55] ter Braack EM, de Vos CC, van Putten MJ. Masking the auditory evoked potential in TMS-EEG: A comparison of various methods. Brain Topogr 2015;28(3):520-8.

[56] Conde V, Tomasevic L, Akopian I, Stanek K, Saturnino GB, Thielscher A, et al. The non-transcranial TMS-evoked potential is an inherent source of ambiguity in TMS-EEG studies. NeuroImage 2019;185:300-12.

[57] Ruohonen J, Ollikainen M, Nikouline V, Virtanen J, Ilmoniemi RJ. Coil design for real and sham transcranial magnetic stimulation. IEEE Trans Biomed Eng 2000;47(2):145-8.

[58] Rossi S, Ferro M, Cincotta M, Ulivelli M, Bartalini S, Miniussi C, et al. A real electro-magnetic placebo (REMP) device for sham transcranial magnetic stimulation (TMS). Clin Neurophysiol 2007;118(3):709-16.

[59] Hoeft F, Wu DA, Hernandez A, Glover GH, Shimojo S. Electronically switchable sham transcranial magnetic stimulation (TMS) system. PloS one 2008;3(4):e1923.

[60] Belardinelli P, Biabani M, Blumberger DM, Bortoletto M, Casarotto S, David O, et al. Reproducibility in TMS-EEG studies: A call for data sharing, standard procedures and effective experimental control. Brain Stimul 2019.

[61] Kawano T, Hattori N, Uno Y, Kitajo K, Hatakenaka M, Yagura H, et al. Large-scale phase synchrony reflects clinical status after stroke: An EEG study. Neurorehabil Neural Repair 2017;31(6):561-70. 